\title{
In-Silico Analysis of Interacting Pathways Through Kim-1 Protein Interaction in Diabetic Nephropathy:
}

Fatima Abid ( $\sim$ Fatima.abid@jsmu.edu.pk)

Jinnah Sindh Medical University

Zil e Rubab Rubab

Ziauddin University

Syeda Sadia Fatima

Aga Khan University Hospital

Aijaz Ahmed Qureshi

Jinnah Sindh Medical University

Arfa Azhar

Aga Khan University Hospital

Aliya Jafri

Jinnah Sindh Medical University

\section{Research Article}

Keywords: KIM1, HAVCR1, Diabetic Nephropathy, Differentially expressed gene, Hub gene, and Immune pathways

Posted Date: September 8th, 2021

DOI: https://doi.org/10.21203/rs.3.rs-738168/v1

License: (c) (i) This work is licensed under a Creative Commons Attribution 4.0 International License. Read Full License 


\section{Abstract}

Human Kidney Injury Molecule-1 also known as HAVCR-1 (Hepatitis A virus cellular receptor 1) belongs to cell-surface protein of immunoglobulin superfamily involved in phagocytosis by acting as scavenger receptor epithelial cells. The study focused to pinpoint the mechanisms and gene that interact with KIM-1.

Methods: This in-silico study was done from March, 2019 to December 2019 .First the Enrichment and protein-protein interaction (PPI) network study of the carefully chosen proteins together with diagramed gene data sets was accomplished using FunRich version 3.1.3.it was done to unveil the proteins that may have an effect on the regulation of HAVCR1 or are may be regulated by this protein. These genes were then further considered in pathway analysis to discover the dysregulated pathways in diabetic nephropathy. The long list of differentially expressed genes is meaningless without pathway analysis.

Results: Important pathways that are dysregulated in diabetic nephropathy patients are identified including Immune System (Total = 237, P<0.05), Innate Immune System (Total=140, P<0.05), Cytokine Signaling Immune system (Total = 116, P<0.05), Adaptive Immune System (Total $=85$ ) and Neutrophil degranulation (Total=78).

Conclusion: The top 5 genes that are interacting directly with HIVCR1 include CASP3, CCL2, SPP1, B2M, and TIMP1 with degrees 161, 144, 108, 107, and 105 respectively for Immune system pathways (Innate Immune System, Cytokine Signaling Immune system, Adaptive Immune System and Neutrophil degranulation)

\section{Introduction}

Diabetic nephropathy (DN) is well identified because of its potential to lead to terminal renal disease (ESRD) that commonly occurs due to complications related to diabetes mellitus [1].Generally tubule-interstitial injury commonly occurs in all of prolonged kidney diseases in addition to diabetes. In DN, complex changes in early adaptive renal structural ensues such as tubular and glomerular hypertrophy have been observed along with the accumulation of components of glomerular mesangium extracellular matrix and tubulo-interstitium[2].

In patients, early detection of the tubular lesion can help to effectively manage DN by glycemic control. Further, glomerular involvement is followed mainly by tubular involvement as various tubular enzymes and proteins are noticeable even before serum creatinine level increases and the appearance of micro albuminuria [3]. Moreover, physiological changes in infiltration capacity can be revealed by serum urea nitrogen, creatinine, and urinary protein levels but they do not act as true markers of 'injury' [4].

A HAVCR1 protein with receptors on surface of immunoglobulin superfamily involved in phagocytosis acting as scavenger receptor epithelial cells [5]. There is a six-cysteine immune-globulin like domain in its extracellular portion, consisting mucinlike $\mathrm{O}$ - glycosylated proteins with a threonine/serine and proline-rich and two N-glycosylation sites [6]. KIM-comprises of one transmembrane, an extra, and an intracellular domain [7]. KIM-1 can oxidize lipoproteins and is also involved to identify apoptotic cell surface-specific epitopes phosphatidylserine [8] KIM-1 intercedes dead and necrotic debris engulfment in the injured epithelial tubules [9]. It facilitates phagocytosis by cell surface binding as well as activating internalization but that epithelial cells transforms into semiprofessional phagocytes. It supports to evade exfoliated cells unwanted attachment to fibronectin as well diminishes tubular obstruction and cast development [10]. These characteristics might make KIM-1 kidney injury an ideal biomarker. The main focus of the project is to identify the genes/pathways that interact directly or indirectly with KIM1.

Study used a network based pathway enrichment methodology for detecting KIM-1 associated biological processes. This method was performed based on known pathway network as well as on biological network analysis. Dysregulated pathways were also identified based on pathway network as well as target network analysis to elaborate the importance of KIM-1 in Diabetic nephropathy. 


\section{Methodology}

This is an in-silico study led between March,2019 to December 2019 .Enrichment and protein-protein interaction (PPI) network exploration of the notorious proteins alongside beside mapped gene data sets was executed using FunRich version 3.1.3.

This study was followed by a study done by one of the authors on KIM-1[11]. Kidney Injury Molecule-1 (KIM-1) peptide in blood is specific to tubular hurt. It assessed kidney injury molecule-1level in diabetics with history of kidney disease and with no history and evaluated screening accuracy of KIM-1. This was a prospective study design that comprised $n=85$ subjects as duplicate samples from the diabetic unit of Jinnah Post Graduate Medical Center and Nephrology department in cooperation with Aga Khan University hospital from November 2016 till September 2017.it was approved by ethical evaluation board of Basic Medical Sciences Institute, JPMC Karachi (Ref NO.F.2-81-IRB/2017/GENL/419/JPMC), Pakistan.

\section{Gene expression data}

In this study, the E-GEOD-30529 gene expression profile 9[12] was obtained from the Array Express database [13], which is based on the Affymetrix Gene Chip Human Genome U133A 2.0. The datasets of E-GEOD-30529 consist of 22 samples comprise of 10 Diabetic Kidney Disease patients whereas the remaining 12 samples are associated with normal conditions.

\section{Data pre-processing and detection of DEG}

Series matrix of the dataset with accession no E-GEOD is downloaded from geo and is processed to convert to .xlsx file as SAM accepts the input file in this format. The DEG was identified through the SAM (Significant Analysis of microarray) method [14]. The genes with delta value 1.27 and fold change

1.35 are considered as differentially expressed. 2729 differentially expressed genes are identified from which top 2000 genes are considered for further analysis.

\section{Construction of the target network}

DEG (Differentially expressed genes) pair's possible functional relationships were investigated through the search tool STRING database for retrieving the interacting genes/ proteins [15]. This network was constructed by giving the DEGs as input to STRING for the Retrieval of Interactions among these genes. The list of genes and their interactions was extracted and used to run a network analysis

\section{Network analysis of topological network and extraction of the target network}

Cytoscape 3.8.2, a free software tool was used for analyzing, modeling, and visualizing the interactions network on molecular and genetic levels [16]. In this article, Cytoscape 3.8.2 was used to characterize the biological importance of genes over the index of topological centrality. Each gene degree quantified the local topology by adding the adjacent genes numbers. It gives a single count of a given node through the number of interactions. The high degree node suggests a central node in the network of interactions [17]. If a degree associated with a particular gene was ranked in the top 5 among the genes in the target network the gene was considered as hub gene.

\section{Construction of pathway network}

STRING is an online application devoted to protein-protein interactions. It is comprising physical (direct) and functional (indirect) interactions. It contains information from publically available databases, experimental sources, co-expression, and genomic perspectives. In this database, 24'584'628 proteins from 5'090 organisms were included at this time [15]. REACTOME database was used to obtain the biological pathways data [18]. In this database, a total of 2441 pathways were included. According to the pathways genes, each pathway was extracted based on the gene pairs from the total gene pair.

\section{Results}




\section{Identification of differentially expressed genes:}

Statistically significant genes in diabetic nephropathy are discovered using the SAM technique. About 2571 genes are discovered as differentially expressed 1765 genes and are up regulated in the diseased as compared to control whereas 805 genes are down- regulated in the diseased group. HIVCR1 is positively differentially expressed with delta value 2.544952 and fold changes value of 1.353374 . Top 2000 genes are considered for further analysis.

\section{Construction of Topological Network:}

2000 genes are given as input to String to create an interaction network. The string network consists of 21975 edges and 1634 nodes is retrieved with the confidence of 0.400 .

\section{Network Analysis and selection of target network:}

The interaction file is later imported to Cytoscape network analysis. Network analysis is performed to compute the topological parameters of the networks. After performing the network analysis only the genes interacting directly or indirectly with the gene of interest i.e. HAVCR1 are selected. The selection is completed in two steps. At the first point, the nodes and edges which are directly connected to HAVCR1 are selected. In the next step, the nodes and edges connected to the first neighbors are selected. As we cannot analyze the complete network obtained from STRING so here only the nodes connected directly or indirectly to our gene of interest is extracted.

Table 1: HAVCR1 and its neighboring genes

\begin{tabular}{|ll|}
\hline Gene & Degrees \\
\hline HAVCR1 & 10 \\
\hline CCL2 & 144 \\
\hline LCN2 & 40 \\
\hline CLU & 66 \\
\hline TIMP1 & 105 \\
\hline IL18 & 90 \\
\hline C3AR1 & 103 \\
\hline B2M & 107 \\
\hline CASP3 & 161 \\
\hline SPP1 & 108 \\
\hline HPGDS & 45 \\
\hline
\end{tabular}

The top 5 genes that are interacting directly with HIVCR1 include CASP3, CCL2, SPP1, B2M, and TIMP1 with degrees 161, 144, 108,107 , and 105 respectively. The top ten genes along with their genes are displayed in Table1. About 450 genes are identified which are interacting directly or indirectly with the protein of interest. The topological network of HAVCR1 along with its neighboring genes is shown in Figure 1. The number of edges in the target network is 941 and the number of nodes is 941

Pathway analysis: 
Using pathway analysis, 85 pathways enriched in differentially expressed genes with $P$ Value $<0.05$ were found. Out of these 26 pathways have neighboring genes later on the number of neighboring genes is calculated by taking the intersection of genes in the pathway and the neighboring genes. Top 5 pathways include Innate and Adaptive Immune System, Neutrophil degranulation, and Cytokine Signaling in Immune system, having neighboring gene count of 237, 147, 85, 78 and 116 respectively. Top 10 pathways along with the number of neighboring genes are shown in Table 2.

Table 2: Major deregulated pathways and genes involved 


\begin{tabular}{|ll}
\hline Pathways & $\begin{array}{l}\text { No of } \\
\text { hub } \\
\text { genes }\end{array}$ \\
\end{tabular}

\section{Involved}

Immune

\section{System}

,QSOX1,RHOA, CFD, VEGFA,FN1, BCL2, CD44,ITPR3,
FCGR2A, VCAM1,PAK2, LCK, ACTB,
SYK,HCK,LYN,ITGB1,VAV1, CYFIP1, ATIC, CCL2, CCR2,
CXCL8,CCL19, CCL5, CXCL1,ANXA1,CCL20, FCGR2B,
CASP8, LAMP2, LPCAT1, CASP3, VCL, COL1A1, IRF1,
TLR2, ITGAV, SAMHD1, ICAM1,TLR3, COL1A2, PDIA3
,CD28, P4HB, CASP9, HMGB1, ANXA2,TLR7, CD63,
KRT8, STAT1, CYBB, ALOX5,ITGAM,PTEN,VIM,
SQSTM1, PTPRC, PECAM1, MX1

ALOX5,ITGAM,PTEN, VIM, SQSTM1, PTPRC, PECAM1, MX1,PPIA, HSP90AB1, RPLPO, MYC, YWHAZ, ITGB3, IRF2, ILF2, ITGB2,CAPN1,CD59, HLA-DQB2, ,C3, PYCARD, AGA,CD3D, IL TR, PANX1,CDA, ADAM10, STK10,CMTM6, MICB, SNAP25, MYD88,ADAM17, FCER1G, LILRB2, CKAP4, C3AR1,CD36,RAP2B, CD53, CD47, TNFRSF1B, PRKCB,SELL, COL3A1, CTSK, IL 18, BST2, HLA-F,IRF8, DDX58,HLA-G, HLA-E, HLA-A,HLA-C,HLA-B, GBP2, IFITM3, PSMB8, OAS1, IRF9, GNB5, PSAP, FPR1, CD14, EDA, RAB27A, NLRP3, XRCC5, CLEC7A, JAK1, TYROBP, CD86, NCF2, LCP2, C1QB, CTSS, LY86, CSF2RB, CSTB, MID1, CD209, SLPI, GZMB,

TNFSF10,EPO,BIRC3, IL 7,CSF1R,CD1D, ATG12,CASP4, IL 15, CASP1, JAK2, CFLAR,APAF1, FGL2, LY96,CD74, SEC24D, SEC24C, SIGLEC1, BCL 10, CARD8, TRIM5, TRIM22,DDOST, SKP2, CDC27, IL 1ORA, DOCK2, PRKDC, GAB2, MAP2K4, QPCT, TXNIP,RET,IKBKB,NFATC1, LTF,TNFAIP6, LILRA2, SP100, TAP1, TRIM38, PSMB10, CD247, TLR1, CD1C, FCGR1B, GBP1, LCN2, PSMB9, NEU1, LAT, TAPBP, TNFAIP3, TRAF3,CYLD, C7,IL11, TNFRSF11B, IFI16, GLIPR1, CPNE1,C1QA, C2,C4BPA,FCGR3B, MRC1, CLEC10A,IL 10RB, IFNGR1, MEF2C, IL33, EIF4G3,

\section{IL1R1,IL32, FNTA, TEK,KLRB1,INPP5D}

\section{Innate}

Immune

System
140

CTSD , LYZ ,SERPING1,CLU ,C1R ,CFH ,CFB ,C1S ,B2M ,QSOX1 , RHOA , CFD

,BCL2, CD44, ITPR3 , FCGR2A,PAK2 , LCK, ACTB, SYK, HCK, LYN, VAV1, CYFIP1,ATIC, CCR2, CXCL 1, FCGR2B, CASP8, LAMP2, LPCAT1, VCL ,TLR2, ITGAV , TLR3 , CASP9, HMGB1 , ANXA2, TLR7, CD63 , KRT8, CYBB , ALOX5, ITGAM , PTPRC , PECAM1 , PPIA , HSP9OAB1, ILF2, ITGB2, CAPN1, CD59, C3

,PYCARD , AGA ,CD3D ,PANX1 , CDA ,ADAM10, STK10, CMTM6, SNAP25, MYD88, FCER1G , LILRB2, CKAP4, C3AR1, CD36, RAP2B, CD53, CD47, TNFRSF1B ,SELL , CTSK , BST2 , DDX58, HLA-E, HLA-A, HLA-C , HLA-B ,PSMB8

, PSAP, FPR1 , CD14, RAB27A, NLRP3 , XRCC5, CLEC7A,TYROBP, NCF2, LCP2, C1QB , CTSS, LY86, CSTB , CD209, SLPI, GZMB , EPO , BIRC3, ATG12, CASP4, CASP1 , CFLAR, APAF1, FGL2, LY96, BCL 10, CARD8, DDOST, DOCK2, PRKDC, GAB2 , MAP2K4 , QPCT , TXNIP, IKBKB , NFATC1 , LTF ,TNFAIP6, PSMB10, CD247, TLR1 , LCN2 , PSMB9, NEU1, LAT ,TNFAIP3,TRAF3 , CYLD , C7, IFI16, GLIPR1, CPNE1 , C1QA , C2, C4BPA , FCGR3B, CLEC1OA,MEF2C 


\section{Cytokine \\ Signaling in \\ Immune \\ system}

SYK, HCK,LYN, ITGB1,VAV1, CCL2, CCR2, CXCL8, CCL19,CCL5, CXCL1, ANXA1

,CCL20,CASP3,VCL, IRF1, SAMHD1, ICAM1,COL1A2, P4HB, HMGB1,ANXA2, STAT1, ALOX5,ITGAM, VIM, SQSTM1, MX1,PPIA, RPLPO, MYC, YWHAZ,ITGB3, IRF2, ITGB2,HLA-DQB2, HLA-DPA1,HLA-DPB1, HLA-DQB1, HLA-DRA,IL 7R, SNAP25, MYD88, ADAM17, CD36, TNFRSF1B,IL 18, BST2, HLA-F,IRF8,DDX58, GBP2,IFITM3, PSMB8,OAS1, IRF9, FPR1, EDA, JAK1,CD86, CSF2RB, MID1, GZMB, TNFSF10, BIRC3,IL7, CSF1R,IL 15, CASP1,JAK2, TRIM5,TRIM22, IL 1ORA ,GAB2, MAP2K4, $R E T$,IKBKB, SP100,TRIM38, PSMB10,

FCGR1B, GBP1, LCN2, PSMB9, LAT,TRAF3,IL11, TNFRSF11B, IL 10RB, IFNGR1, MEF2C,IL33, EIF4G3, IL 1R1,IL32, FNTA, TEK,INPP5D

$\begin{array}{lll}\text { Adaptive } & 85 & \text { CTSD, HSPA5, B2M ,ITPR3, VCAM1, PAK2, LCK, SYK, LYN, ITGB1, VAV1, } \\ \text { FCGR2B, COL1A1, TLR2, ITGAV, ICAM1, COL 1A2, PDIA3, CD28, CYBB, PTEN }\end{array}$

Immune System
, PTPRC, PPIA , YWHAZ,ITGB2, HLA-DQB2,HLA-DPA1,HLA-DPB1,HLA-DMB, HLADMA,HLA-DQB1,HLA-DRA,C3 ,CD3D , MICB , SNAP25, MYD88, LILRB2 , CD36, PRKCB ,SELL , COL3A1, CTSK, HLA-F , PSMB8, GNB5, FPR1 , CD14, TYROBP , CD86, NCF2 ,LCP2 , CTSS, CD209, TNFSF10,CD1D , LY96, CD74 , SEC24D, SEC24C, SIGLEC1,BCL 10, SKP2,

CDC27, IKBKB, NFATC1 , LILRA2,TAP1 , PSMB10 , CD247, TLR1 , CD1C , FCGR1B , PSMB9 , LAT , TAPBP , MRC1 , IFNGR1 , KLRB1 , INPP5D,
CTSD , LYZ ,C1R ,B2M ,QSOX1 , RHOA , CFD ,CD44 , FCGR2A , CYFIP1 , ATIC , CXCL 1, LAMP2, LPCAT1, VCL ,TLR2, ITGAV, HMGB1, ANXA2, CD63, KRT8, CYBB , ALOX5 , ITGAM ,PTPRC, PECAM1 , PPIA , HSP9OAB1,ILF2 , ITGB2 , CAPN1

, CD59,C3 ,PYCARD , AGA ,CDA ,ADAM10, STK10, CMTM6, SNAP25,FCER1G, LILRB2 , CKAP4 , C3AR1 , CD36, RAP2B , CD53, CD47, TNFRSF1B, SELL , BST2 , PSAP, FPR1,CD14, RAB27A, XRCC5, TYROBP , CTSS, CSTB, SLPI, GZMB, EPO , APAF1 , FGL2 , DDOST, DOCK2 , QPCT , LTF ,TNFAIP6, LCN2 , NEU1, GLIPR1, CPNE1, CABPA , FCGR3B

\section{Signaling by 69 Interleukins}

TIMP1 , HGF ,VEGFA , FN1 ,BCL2, VCAM1, PAK2 , LCK, SYK,HCK, LYN ,ITGB1, VAV1, CCL2, CCR2, CXCL8,CCL 19, CCL5, CXCL 1, ANXA1, CCL20,CASP3, ICAM1, COL 1A2, P4HB, HMGB1, ANXA2, STAT1 , ALOX5, ITGAM, VIM, SQSTM1, PPIA, RPLPO , MYC, YWHAZ, ITGB2 , IL 7R, SNAP25, MYD88, CD36, TNFRSF1B,IL 18, PSMB8, FPR1, JAK1, CD86, CSF2RB, GZMB , IL7, CSF1R, IL 15 , CASP1, JAK2 , IL 1ORA , GAB2 ,MAP2K4 , IKBKB , PSMB10, LCN2 , PSMB9, IL 11,

IL 10RB, IFNGR1, MEF2C, IL33, IL 1R1 , IL32 , INPP5D,

A2M ,TIMP1, SPARC, CTSD, MMP7, THBS1, VWF ,ACTN1 ,TGFB2, FN1 ,CD44, VCAM1, ITGB1 , COMP , CASP3 , ITGB8, COL 1A1, ITGAV , ICAM1, ITGA2, COL1A2, P4HB , SPP1, ITGAM ,PECAM1 , ITGB3 , ADAM12, ITGB2 , CAPN1, ADAM10, LAMB1, LAMC1,ADAMTS5,ADAMTS3,ADAM17, TNC,SDC2, VCÁN, MATN3 , CD47, FBN1 , COL5A1, MMD, DCN ,FMOD , BGN,COL3A1, LUM ,CTSK, BMP7, CTSS, GZMB , CAST, SERPINH1,CAPN6, LAMC2 ,IKBKB , NID1 , COL $4 A 1$,' $\angle T B P 3$,

Platelet activation, $\quad 52$ signaling and aggregation
A2M ,TIMP1 , HGF ,SPARC, HRG ,SRGN , THBS1, VWF ,KNG1 , EGF ,SERPING1, CLU, HSPA5, TOR4A, QSOX1, RHOA, TMSB4X, CFD ,ACTN1, VEGFA , F5 ,TGFB2 , FN1 ,ITPR3 ,LCK,SYK, LYN, VAV1 , LAMP2, VCL ,COL1A1 , ANXA5 ,COL1A2 ,CD63, PECAM1 , PPIA , YWHAZ, ITGB3 , PLEK, FCER1G, CALU, CD36, $P R K C B, G N B 3$, GNB5, ADRA2A, PSAP, GNB1, PLA2G4A,LCP2, LAT ,LGALS3BP

\section{Discussion}

Pathogenesis of DN is complex, and renal pathological lesions are diverse. Diagnosis and progression of diabetic nephropathy (DN) has long been monitored through Glomerular filtration rate (GFR) which was the best functional marker of kidney. But it 
was time taking, and it typically calculates from equations that rest on on serum creatinine and cystatin C. Micro albuminuria and proteinuria have been another noninvasive biomarker but their limitations include that patients having advanced kidney disease and microalbuminuria detected in them do not respond to therapy as it would be at an earlier stages. Further; the ultrafiltrated Proteins cause advanced level of tubulo-interstitial damage to the kidney $[19,20]$ Diabetic nephropathy embraces events causing to destruction of filtration membrane, capillaries and causing the kidney function to be impaired [21]

Additional disparities consist of: mesangial cell Expansion, Kimmelstiel-Wilson nodules, glomerulosclerosis, and tubular Interstitial fibrosis [22] reason behind due to both hereditary plus ecological factors linked with diabetes, the most noteworthy being High blood glucose and high blood pressure. Primary functional irregularity is intra-glomerular hypertension, Hyper filtration and supplemented by the onset of Micro albuminuria [23]. The cellular components of the kidney react to hyperglycemia by several ways: hemodynamic, metabolic, and Inflammatory [24, 25]. There are three pathways involved including

Hemodynamic Pathway which is based on the fact that because of initiation of the renin-Angiotensin-aldosterone-mechanism in which efferent arteriolar constriction Which culminates in the raised pressure of intra-glomerular blood vessels as well as Hyper filtration [25] The renin-angiotensin-Aldosterone-system is activated by hyperglycemia in diabetic patients. The Angiotensin II is responsible for initiation of inflammation, renal cell growth and Self-digestion/ degradation of podocytes [23] Glomerular perfusion and glomerular filtration rate (GFR) which are Normally kept in pace by an auto regulatory mechanism are also deranged and Perturbed by the prolonged elevated glucose levels. This process, although initially Increases in GFR but later on leads to thickening of endothelial mesengial cells [21]

Other main pathway is Metabolic Pathway it contributes to pathogenesis of diabetic nephropathy by excessive glucose degradation among cells - a process called glycolysis. This enhances the polyol pathway in which there is excessive formation and storing of Sorbitol in cells. Sorbitol lowers the level of nitric oxide thus leading to Intracellular stress and apoptosis. Next to this impact is formation of fructose from Sorbitol which is highly nephrotoxic. Fructose directly causes loss of protein from Kidneys, lowers the glomerular filtration rate and abundance of superoxide's ions And other inflammatory cytokines [25] second to this is hexosamine pathway stems which is by product of Glycolysis. Tumor necrosis factor-a and transforming growth factor-B1. All this ends in Hypertrophy of nephrocytes [25] Advanced glycation end-products which are reason of enhancing of vascular complication of diabetes mellitus, are shaped through the advanced Glycation of proteins in the renal cells. These products when accumulate around the Tubulointersitial cells and glomeruli in the kidneys, further lead to hardening of the Glomerular basement membrane and fibrosis of these structures [23]

Advanced glycation end-products also are capable of attaching to pro-Inflammatory receptors; altering the actions of renal cells also Give rise to cytokines and reactive oxygen species (ROS) which are Nephrotoxic [25].The last step of glucose degradation in which there is stimulation of Protein kinase $\mathrm{C}(\mathrm{PKC})$ is formed form glyceraldehyde-3-phosphate. This Production of PKC directly leads to initiation two vasodilator substances;

Prostaglandin E2 and nitric oxide (NO) [25] this lead to increased Glomerular filtration as said Earlier. It also been documented that activated protein kinase $\mathrm{C}$ leads to glomerular Basement membrane stiffening and buildup of extracellular matrix which Both affect permeability of capillaries and in turn much amount of albumin enter the vessels [23]Lastly the most important and contributing pathway related and justified by our analysis is Inflammatory Immune Pathway. The chronicity of diabetes mellitus leads to inflammatory signals which affect the renal cells and cause modification and remodeling as well as triggering the innate and adaptive Immune response [26,27]. Together all these and releasing inflammatory cytokines engender the enhanced glomerular basement membrane thickening, fibrosis and increased permeability of capillaries [28]Kidney damage as a result of high blood glucose levels in diabetic patients is known as diabetic nephropathy. The expression level of KIM1 along with AER (albumin excretion rate) can act as a diabetic nephropathy prognostic marker [29]. Many studies show that KIM1 can act as a diabetic nephropathy prognostic marker. The purpose of carrying this research is the identification of deregulated pathways through KIM1 interaction. 
Many diabetic patients are observed to suffer from kidney-related diseases and inflammation. In several experimental studies, CCL2 is proposed as a potential healing target and biomarker in renal tissue impaired patients having diabetes [30]. $L C N 2$ is predicted as an early significant biomarker to diagnose the $\mathrm{DN}$ at early and developing stages [32]. CLU is experimentally observed to be overexpressed in DN, thus predicted as a therapeutic target and potential biomarker of DN. TIMP1 is as well experiential to show an important part in the advancement of DN due to its under-expression [33]. IL 18 (interleukin-18) has a significant role as an inflammation mediator in the development and progression of NP. IL 18 is reported to be overexpressed in renal tissues in Diabetic Nephropathy [34]

C3AR1 (Complement C3a Receptor 1) is involved in inflammatory responses and is overexpressed in diabetic patients. It is experimentally demonstrated in a study that knockout of C3AR1 in mice, slows down the progression of (Diabetic Nephropathy) DN [35]. B2M (beta-2- microglobulin) is observed to be overexpressed in kidney-related diseases. It is reported as an early biomarker in DN development [36]. CASP3 is proposed as a significant therapeutic target in DN. The inhibition of CASP3 in mice resulted in exacerbating symptoms of DN. Consequently, it could be treated as a potential therapeutic target [35]. SPP1 is experimentally reported as differentially excreted protein in DN patients [36].

\section{Conclusion}

In this exploration, crucial mechanisms were brought to focus using protein-protein interaction of network, centered on The top 5 genes that are interacting directly with HIVCR1 include CASP3, CCL2, SPP1, B2M, and TIMP1, correspondingly for Immune system pathways (Innate Immune System, Cytokine Signaling Immune system, Adaptive Immune System pathways and Neutrophil degranulation)

Were established to be amplified in Diabetic nephropathy through Protein Interaction studies. These signaling pathways and associated proteins serve to be potential target for novel beneficial agents to decrease the burden of Diabetic nephropathy resulting from chronic diabetes mellitus.

\section{Declarations}

\section{Ethics approval and Consent to participate}

All procedures were in accordance with the ethical standards of Helsinki's declaration after approved by ethical committee of Basic Medical Sciences

Institute, JPMC Karachi, Pakistan NO.F.2-81-IRB/2017/GENL/419/JPMC), Pakistan.

The written and verbal consents were obtained for the research.

Availability of Data and material: Not Applicable

Competing interest: The authors declare that they have no conflict of interest.

Funding: Not Applicable

Author's contribution:

FA did the literature review, Computational analysis and manuscript writing, ZR designed the methodology and reviewed the manuscript contributed in critical review, AQ assisted in research write up, AA helped in Analysis and editing, AJ performed the review of manuscript. All authors approved the final manuscript for publication.

Acknowledgements: Not Applicable

\section{References}

Page $9 / 11$ 
1. M. Sugahara, T. Tanaka, R. Inagi, and M. Nangaku, “Diabetic kidney disease," in Diabetes and Aging-related Complications, 2017.

2. K. Reidy, H. M. Kang, T. Hostetter, and K. Susztak, "Molecular mechanisms of Diabetic kidney disease," Journal of Clinical Investigation. 2014.

3. D. Shan, H. M. Wu, Q. Y. Yuan, J. Li, R. Le Zhou, and G. J. Liu, “Pentoxifylline for diabetic kidney disease,” Cochrane Database of Systematic Reviews. 2012.

4. A. K. H. Lim, “Diabetic nephropathy - Complications and treatment," Int. J. Nephrol. Renovasc. Dis., 2014.

5. W. Huo, K. Zhang, Z. Nie, Q. Li, and F. Jin, “Kidney injury molecule-1 (KIM-1): a novel kidney-specific injury molecule playing potential double edged functions in kidney injury, "Transplantation Reviews. 2010.

6. J. V. Bonventre and L. Yang, "Kidney injury molecule-1," Current Opinion in Critical Care. 2010.

7. J. Mishra et al., “Kidney NGAL is a novel early marker of acute injury following transplantation,” Pediatr. Nephrol., 2006.

8. T. Ichimura, E. J. P. V. Asseldonk, B. D. Humphreys, L. Gunaratnam, J. S. Duffield, and J. V. Bonventre, "Kidney injury molecule-1 is a phosphatidylserine receptor that confers a phagocytic phenotype on epithelial cells," J. Clin. Invest., 2008.

9. V. S. Sabbisetti et al., "Blood kidney injury molecule-1 is a biomarker of acute and chronic kidney injury and predicts progression to ESRD in type I diabetes," J. Am. Soc. Nephrol., 2014.

10. L. Yang et al., "KIM-1-mediated phagocytosis reduces acute injury to the kidney," J. Clin. Invest., 2015.

11. Khan FA, Fatima SS, Khan GM, Shahid S. Evaluation of kidney injury molecule-1 as a disease progression biomarker in diabetic nephropathy. Pak J Med Sci. 2019;35(4):992-996. Doi: https://doi.org/10.12669/pjms.35.4.

12. N. Kolesnikov et al., "arrayexpress update-simplifying data submissions," Nucleic Acids Res., 2015.

13. V. G. Tusher, R. Tibshirani, and G. Chu, "Significance analysis of microarrays applied to the ionizing radiation response," Proc. Natl. Acad. Sci. U. S. A., 2001.

14. D. Szklarczyk et al., "STRING v11: Protein-protein association networks with increased coverage, supporting functional discovery in genome-wide experimental datasets," Nucleic Acids Res., 2019.

15. D. Otasek, J. H. Morris, J. Bouças, A. R. Pico, and B. Demchak, "Cytoscape Automation: Empowering workflow-based network analysis," Genome Biol., 2019.

16. N. T. Doncheva, J. H. Morris, J. Gorodkin, and L. J. Jensen, “Cytoscape stringapp: Network Analysis and Visualization of Proteomics Data," J. Proteome Res., 2019.

17. A. Fabregat et al., "The Reactome Pathway Knowledgebase," Nucleic Acids Res., 2018.

\section{Figures}




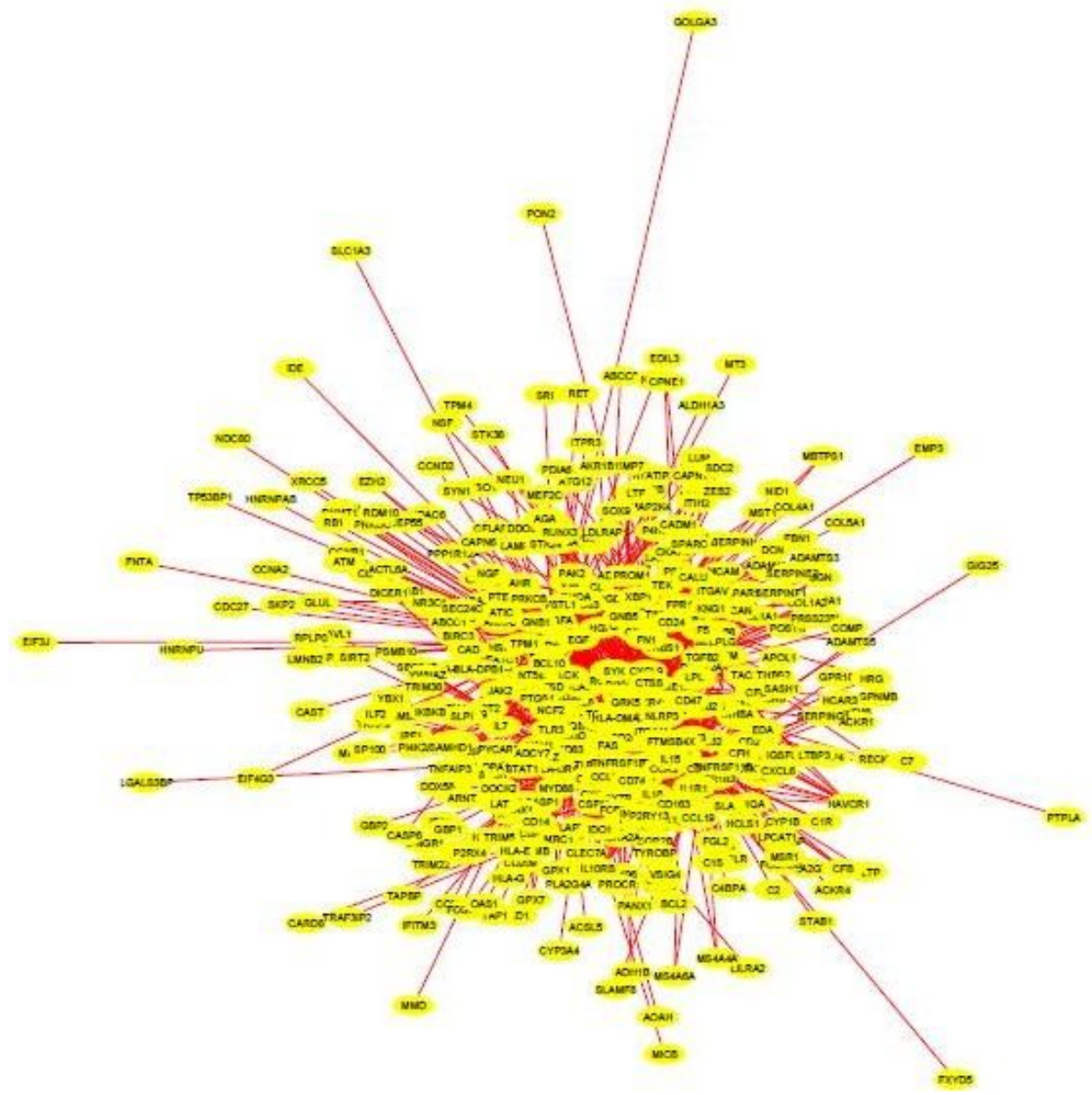

Figure 1

Target network comprising of HAVCR1 and its neighbors

\section{Supplementary Files}

This is a list of supplementary files associated with this preprint. Click to download.

- IRBSUPPLEMENTALFILE.docx 